\title{
SPECIALIZATIONS IN DIFFERENTIAL ALGEBRA
}

\author{
BY \\ AZRIEL ROSENFELD \\ INTRODUCTION
}

1. Objectives and summary. Much of elementary differential algebra can be regarded as a generalization of the algebraic geometry of polynomial rings over a field to an analogous theory for rings of differential polynomials (d.p.) over a differential field $\left({ }^{1}\right)$. To date, however, considerable parts of basic algebraic geometry have yet to be "lifted" into differential algebra. The purpose of the present paper is to fill one such conspicuous gap by developing fundamental parts of a theory of specializations and dimensions over differential fields.

Chapter I is devoted to certain necessary preliminaries. Among the concepts introduced is a useful weakening of the notion of reducedness of d.p. In terms of this, a type of set of d.p., called a coherent autoreduced set, is defined, for which a certain close relationship holds between the ideal and the differential ideal (d.i.) generated by the set. Coherent autoreduced sets of d.p. figure centrally in the proofs of the main theorems, since it turns out that their use enables one to reduce these theorems to analogous theorems for suitable polynomial rings.

Chapter II contains the proofs of two basic theorems on extensions of specializations over differential fields. Roughly stated, these are:

(1) Any specialization not annihilating a certain d.p. can be extended to a specialization not annihilating a given d.p. $\left({ }^{2}\right)$.

(2) If certain "properness" conditions hold, any intermediate specialization of parametric indeterminates can be extended to an intermediate specialization.

In Chapter III some applications of the above results are given. First among these are three propositions on the constructibility of ascending and descending chains of prime d.i. between various bounds. Second is a theorem concerning the dimensions of certain "nonsingular" prime d.i. components of a coherent autoreduced set; this theorem provides a partial answer to a ques-

Received by the editors May 13, 1957.

(1) For the basic terminology and theorems of differential algebra see Ritt [6, Chapter I, pp. 1-14, Chapter II, pp. 21-23, 26-28, 33-34 and the parallels in Chapter IX]; Kolchin [3, pp. 23-26]; and Kolchin [4, pp. 761-771]. An acquaintance on the part of the reader with this material will be assumed.

(2) The theorem referred to here was proved for ordinary differential fields by Ritt (see Ritt [5, pp. 543-545]). A proof using elimination-theoretic methods has just (late 1956) appeared in Seidenberg [8]. 
tion posed by Ritt (see Ritt [6, p. 178]). Finally, an indication is given of how these results furnish the first steps toward the development of a theory of the dimensions of the components of the intersection of two algebraic differential manifolds (in this paper called "varieties").

The author wishes to express his indebtedness to Dr. Ellis R. Kolchin for his many helpful suggestions concerning the present paper.

2. Open questions. A number of the theorems in this paper are proved only on the basis of "properness" or "nonsingularity" hypotheses, which turn out to be conditions of the form: The initials and separants of certain d.p. do not vanish at a certain point. These particular hypotheses have to be introduced on account of the nature of the methods of proof being usedfor example, on account of our use of the process of reduction of d.p. Examples show, furthermore, that some sort of hypotheses of "nonsingularity" must be required, since the theorems in question do not hold unrestrictedly. However, other examples show that the particular hypotheses actually used here are by no means necessary conditions for the correctness of the respective theorems. Thus the problem is posed: Can analogous conditions be formulated which are both necessary and sufficient?

\section{I: Preparations}

1. Some notation and terminology. Throughout what follows, $F$ will denote a fixed differential field of characteristic zero; the derivations under which $\mathcal{F}$ is a differential field will be denoted by $\delta_{1}, \cdots, \delta_{m}$. All "points" or "zeros" which come under consideration are understood to have their coordinates in a preselected universal extension $\Omega$ of $\mathcal{F}$.

If $\delta$ is any subset of $\Omega$, we denote by $\mathcal{F}[\mathcal{S}], \mathcal{F}(\mathcal{S}), \mathcal{F}\{\delta\}$ and $\mathcal{F}\langle\mathcal{S}\rangle$ respectively the ring, the field, the differential ring and the differential field generated in $\Omega$ by adjoining $\mathcal{S}$ to $\mathcal{F}$. (It will be recalled that if $\overline{\mathcal{S}}$ is the closure of $\mathcal{S}$ in $\Omega$ under the $\delta$ 's, then $\mathcal{F}\{\delta\}=\mathcal{F}[\bar{\delta}]$ and $\mathcal{F}\langle\mathcal{S}\rangle=\mathcal{F}(\overline{\mathcal{S}})$.)

If $R$ is any differential ring and $\Sigma$ any subset of $R$, we denote by $(\Sigma)$, $\langle\Sigma\rangle,[\Sigma]$ and $\{\Sigma\}$ respectively the ideal, the radical ideal, the differential ideal and the perfect differential ideal generated by $\Sigma$ in $R$. (If $\bar{\Sigma}$ is defined analogously to $\bar{\S}$ of the last paragraph, one sees that $[\Sigma]=(\bar{\Sigma})$. Furthermore it can be shown (Ritt $[6$, p. 8]) that in the cases of present interest one also has $\{\Sigma\}=\langle\bar{\Sigma}\rangle$, or $\{\Sigma\}=\langle[\Sigma]\rangle$.)

In what follows, $R$ will always denote the differential polynomial ring $\mathcal{F}\left\{y_{1}, \cdots, y_{n}\right\}$, where the $y$ 's are indeterminates.

By a derivative operator we mean a formal power product $\theta=\delta_{1}^{i_{1}} \cdots \delta_{m}^{i_{m}}$ (where the $i$ 's are non-negative integers); we call $\theta$ proper if $\sum_{j=1}^{m} i_{j}$ (the order of $\theta)$ is positive. If $F \in R$, the expressions of the form $\theta F$ ( $\theta$ a derivative operator) are called $F$-derivatives; in particular, the expressions $\theta y_{i}(1 \leqq i \leqq n)$ are called $\boldsymbol{y}$-derivatives. 
A well-ordering of the $y$-derivatives of $R$, written "higher than," is called a ranking if it satisfies the following two conditions:

(1) $\delta_{j} \theta y_{i}$ is higher than $\theta y_{i}(1 \leqq j \leqq m, 1 \leqq i \leqq n$, all $\theta)$.

(2) $\phi y_{i}$ higher than $\theta y_{h}$ implies $\delta_{j} \phi y_{i}$ higher than $\delta_{j} \theta y_{h}(1 \leqq j \leqq m, 1 \leqq h$, $i \leqq n$, all derivative operators $\theta$ and $\phi$ ).

It can be shown that any total ordering of the $y$-derivatives of $R$ which satisfies (1) and (2) is a ranking. Examples are the "marks" of Riquier (Ritt [6, pp. 151-152]); one sees in fact that an arbitrary ranking has just the properties of a "complete system of marks" required for developing partial differential algebra. In particular, the leader, initial and separant of a d.p., and the notion of reducedness, can all be defined in terms of any ranking $\left({ }^{3}\right)$.

A ranking is called unmixed if it satisfies also

(3) $y_{i}$ higher than $y_{h}$ implies $\phi y_{i}$ higher than $\theta y_{h}(1 \leqq h, i \leqq n$; all $\theta, \phi)$.

By an autoreduced set (Ritt: "chain") we mean a set of d.p. of $R$ such that each member is reduced with respect to every other member. (It can be shown-compare Ritt [6, p. 164] - that such a set must be finite.) We adopt the standard notation $A=A_{1}, \cdots, A_{k}$, where the $A$ 's are written with leaders in order of ascending rank, for an autoreduced set of $\mathbb{R}$. The leader, initial and separant of $A_{i}$ will be denoted respectively by $u_{i}, I_{i}, S_{i}$; we will write for short $I=\prod_{i=1}^{k} I_{i}, S=\prod_{i=1}^{k} S_{i}$. We will use, e.g., the notation " $S^{\infty}$ " when we mean "some (sufficiently high) power of $S$ ".

We call $F \in R$ partially reduced (PR) with respect to $A$ if $F$ is free of proper derivatives of the $u_{i}$. (Thus "reduced" breaks up into "PR" + "of lower degree in $u_{i}$ than $A_{i}(1 \leqq i \leqq k)$ "). In terms of this notion, the reduction algorithm (Ritt [6, pp. 5-7 and p. 165]) can be broken up as follows:

(1) For any $F \in R$ there are defined nonnegative integers $s_{1}, \cdots, s_{k}$, and an $F_{0} \in R, \mathrm{PR}$ with respect to $A$, such that $S_{1}^{s_{1}} \cdots S_{\boldsymbol{k}}^{s_{k}} F \equiv F_{0}$ modulo $[A]$. ( $F_{0}$ is called the partial remainder of $F$ with respect to $A\left({ }^{4}\right)$.)

(2) For any $G \in R, P R$ with respect to $A$, there are defined nonnegative integers $i_{1}, \cdots, i_{k}$, and a $G^{0} \in \mathbb{R}$ reduced with respect to $A$, such that $I_{1}^{i_{1}} \ldots I_{k}^{i_{k}} G \equiv G^{0}$ modulo $(A)$. (If $G$ is the partial remainder $F_{0}$ of $F$ with respect to $A, G^{0}$ is called the remainder of $F$ with respect to $A$; evidently this is the same as the usual definition of "remainder".)

Let $\Omega^{k}$ denote the $k$ th Cartesian power of $\Omega$. The subset $U$ of $\Omega^{n}$ consisting of the zeros of a subset $\Sigma$ of $R$ will be called the variety (Ritt: "algebraic differential manifold") associated with $\Sigma$. As in algebraic geometry, through the use of the Theorem of Zeros (Nullstellensatz) one establishes a one-to-one correspondence between the varieties of $\Omega^{n}$ and the perfect d.i. of $R$. One then further proceeds to establish a correspondence between (union-) irreducible varieties of $\Omega^{n}$, prime d.i. of $R$, and equivalence classes (under "generic

( $\left.{ }^{3}\right)$ Strictly speaking, these are only defined for d.p. which $\in \mathcal{F}$. In what follows, no explicit mention of the exceptions is made; it can be verified that for our purposes they are trivial.

(4) For this process of partial reduction, and through much of $\$ 2$ of this chapter, $A$ could be taken somewhat more generally. 
specialization over $\mathfrak{F}$ ") of points of $\Omega^{n}$. The (irreducible) components of a variety (or, correspondingly: the (prime d.i.) components of a perfect d.i. of $R$ ), and the concepts of order (=transcendence degree) and dimension (=differential transcendence degree), are all defined as usual.

2. A fundamental lemma. Let $\boldsymbol{A}$ be an autoreduced set in $R$. Let $u_{i j}(1 \leqq i, j \leqq k ; i \neq j)$ be the lowest common derivative of $u_{i}$ and $u_{j}$, if such

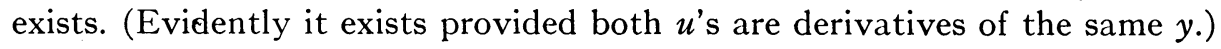
Suppose $\theta_{i j} u_{i}=\theta_{j i} u_{j}=u_{i j}$; let $A_{i j}=\theta_{i j} A_{i}, A_{j i}=\theta_{j i} A_{j}$, and $\Delta_{i j}=S_{j} A_{i j}-S_{i} A_{j i}$. We call $A$ coherent if, for every such pair $i, j,(I S)^{\infty} \Delta_{i j}$ can be written as a linear combination (with coefficients in $R$ ) of $A$-derivatives which have leaders lower than $u_{i j}$ (in rank). (Example. If $\boldsymbol{A}$ is a characteristic set for a d.i. of $R$, then evidently each $\Delta_{i j}$ has remainder zero with respect to $A$; hence $A$ is coherent.)

The fundamental property of coherent autoreduced sets can be stated as follows:

Lemma. $A$ is coheront autoreduced if and only if any $G$ in $[A]:(I S)^{\infty}$ which is $P R$ with respect to $A$ must actually be in $(\boldsymbol{A}):(\boldsymbol{I S})^{\infty}\left(^{(5)}\right.$.

Proof. To see the sufficiency, suppose that the hypothesis on the $\Delta^{\prime}$ s fails to hold for some $\Delta_{i j}$. Then in particular this $\Delta_{i j}$ has remainder $\neq 0$ with respect to $A$. This remainder is of course PR with respect to $A$ and is in $[A]:(I S)^{\infty}$; but by the failure of the hypothesis, it cannot be in $(A):(I S)^{\infty}$, since the $u$ 's themselves are certainly all lower in rank than $u_{i j}$.

We prove the necessity. If $G$ is in $[A]:(I S)^{\infty}$, we can write

$$
(I S)^{\infty} G=\sum_{i=1}^{k} C_{i} A_{i}+\sum_{1 \leqq i \leqq k ; \theta \text { proper }} C_{i, \theta} \theta A_{i} \text { (with the } C^{\prime} \text { 's in } R \text { ). }
$$

Let $\Theta y_{l}=\theta_{i_{1}} u_{i_{1}}=\theta_{i_{2}} u_{i_{2}}=\cdots=\theta_{i_{h}} u_{i_{h}}$ be the highest ranking $y$-derivative effectively present in the right member of the identity (1) as the leader of a proper $A$-derivative.

If $h=1$, make the substitution $\sigma: \Theta y_{l}=\left(S_{i_{1}} \theta_{i_{1}} u_{i_{1}}-\theta_{i_{1}} A_{i_{1}}\right) / S_{i_{1}}$. Since this replaces $\Theta y_{l}$ by something involving only $y$-derivatives lower in rank than itself, it is seen that it transforms (1) into an identity with the same left member, with new $C$ 's in the right member, and with the highest leader of an $A$-derivative effectively present in the right member now lower than $\Theta y_{l}$.

If $h>1$, note first that the group of terms $\sum_{j=1}^{h} C_{i_{j}, \theta i j} \theta_{i_{j}} A_{i_{j}}$ in the right member of (1) can be rewritten in the form

$$
D_{1} \theta_{i_{1}} A_{i_{1}}+\sum_{j=2}^{h} D_{j}\left(S_{i_{j}} \theta_{i_{1}} A_{i_{1}}-S_{i_{1}} \theta_{i_{j}} A_{i_{j}}\right)
$$

(5) The very simple case of this lemma where $\boldsymbol{A}$ is a single d.p. is in Ritt $[6, \mathrm{p} .30]$. An analogous, but apparently somewhat weaker, result is in the recent Seidenberg $[8$, p. 51 , Theorem 6]. 
where

$$
D_{1}=\sum_{j=1}^{h} \frac{S_{i_{j}}}{S_{i_{1}}} C_{i_{j}, \theta_{i j}} ; \quad D_{j}=\frac{-C_{i_{j}, \theta_{i j}}}{S_{i_{1}}} \quad(2 \leqq j \leqq k) .
$$

Now $\theta_{i_{1}} u_{i_{1}}=\theta_{i_{j}} u_{i_{j}}$ implies $\theta_{i_{1}}=\phi_{j} \theta_{i_{1} i_{j}}, \theta_{i_{j}}=\phi_{j} \theta_{i_{j} i_{1}}$ for some (not necessarily proper) derivative operator $\phi_{j}$; this means that $\theta_{i_{1}} A_{i_{1}}=\phi_{j} A_{i_{1} i_{j}}$ and $\theta_{i_{j}} A_{i_{j}}$ $=\phi_{j} A_{i_{j} i_{1}}(2 \leqq j \leqq k)$. We proceed by induction on the order of each of these $\phi_{j}^{\prime}$ 's.

If $\phi_{j}$ is the identity operator, then $S_{i_{j}} \theta_{i_{1}} A_{i_{1}}-S_{i_{1}} \theta_{i_{j}} A_{i_{j}}$ is just $\Delta_{i_{1} i_{j}}$, which by hypothesis on $A$ can be written as a linear combination (over $R$ : $(I S)^{\infty}$ ) of $A$-derivatives with leaders lower in rank than $u_{i_{1} i_{j}}$ (and thus a fortiori lower than $\Theta y_{l}$ ).

On the other hand, if $\phi_{j}$ is proper, we can write it as $\delta_{i} \phi_{j}^{\prime}(1 \leqq i \leqq m)$, and we then have

$$
\begin{aligned}
& S_{i_{j}} \theta_{i_{1}} A_{i_{1}}-S_{i_{1}} \theta_{i_{j}} A_{i_{j}}=S_{i_{j}} \delta_{i} \phi_{j}^{\prime} A_{i_{1} i_{j}}-S_{i_{1}} \delta_{i} \phi_{j}^{\prime} A_{i_{j} i_{1}} \\
& \quad=\delta_{i}\left(S_{i_{j}} \phi_{j}^{\prime} A_{i_{1} i_{j}}-S_{i_{1}} \phi_{j}^{\prime} A_{i_{j} i_{1}}\right)-\left(\delta_{i} S_{i_{j}} \cdot \phi_{j}^{\prime} A_{i_{1} i_{j}}-\delta_{i} S_{i_{1}} \cdot \phi_{j}^{\prime} A_{i_{j} i_{1}}\right) .
\end{aligned}
$$

But since $\phi_{j}^{\prime}$ has order strictly less than that of $\phi_{j}$, by induction hypothesis $S_{i_{j}} \phi_{j}^{\prime} A_{i_{1} i_{j}}-S_{i_{1}} \phi_{j}^{\prime} A_{i_{j} i_{1}}$ can be written as a linear combination (over $R$ : $(I S)^{\infty}$ ) of $A$-derivatives with leaders lower than $\left(\Theta / \delta_{i}\right) y_{l}$, so that the first term of (3) can be written as such a linear combination with leaders lower than $\Theta y_{l}$; and the second term of (3) is already a linear combination of these over $a$.

We have thus shown that for each $j(2 \leqq j \leqq h), S_{i_{j}} \theta_{i_{1}} A_{i_{1}}-S_{i_{1}} \theta_{i_{j}} A_{i_{j}}$ can be written as a linear combination (over $\left.\mathbb{R}:(I S)^{\infty}\right)$ of $A$-derivatives with leaders lower than $\Theta y_{l}$. If we apply this result to (2), we see that it actually enables us to rewrite the right member of (1) so as to leave only one $A$-derivative in it with leader $\Theta y_{l}$ (namely: $\theta_{i_{1}} A_{i_{1}}$ ); in other words, we can rewrite (1) so as to make $h=1$. The leader $\Theta y_{l}$ can now be eliminated entirely by means of the substitution $\sigma$.

In conclusion, then, if we iterate this entire process, lowering the rank of the highest leader of a proper $A$-derivative effectively present in the right member of (1) at each step, we can ultimately reduce (1) to an identity of the form $(I S)^{\infty} G=\sum_{i=1}^{k} E_{i} A_{i}$ (E's in $R$ ); which of course means that $G$ is in $(A):(I S)^{\infty}$, Q.E.D.

The lemma has the following useful corollaries:

(1) If $A$ is coherent autoreduced, then for any $F$ in $\{A\}: I S$, the partial remainder of $F$ with respect to $A$ is in $\langle A\rangle: I S$.

(2) If $A$ is coherent autoreduced, then for any $F$ in $R$ PR with respect to $A$, if $A$ considered as a set of polynomials (in $\mathcal{F}\left[\cdots \theta y_{i} \cdots\right]$ ) has a zero which does not annihilate $I S F$, then even considered as a set of differential polynomials (in $R$ ) $A$ has a zero which does not annihilate $I S F{ }^{6}$ ). (A zero of

$\left(^{6}\right)$ Compare Seidenberg [8, p. 52, Theorem 7]. 
a subset $\Sigma$ of $R$ regarded as a polynomial ring will be called an "ordinary" zero of $\Sigma$.)

We close this chapter by mentioning some additional properties of coherent autoreduced sets which indicate how these sets can be used to bridge the gap between $R$ and the "underlying" polynomial ring. (We omit the proofs, which are not difficult.)

(1) If $A$ is coherent autoreduced, then $A$ is a characteristic set for $\{A\}: I S$ if and only if it is a characteristic set for $\langle A\rangle: I S$.

(2) If $A$ is coherent autoreduced, then $\{A\}: I S$ is a prime d.i. if and only if $\langle\boldsymbol{A}\rangle: I S$ is a prime ideal.

The case where $A$ is such that $I S=1$ (i.e.: Where $A$ is an orthonomic set of d.p.; see Ritt [6, Chapter VIII]) is of special interest:

(3) Let $\boldsymbol{A}$ be an orthonomic autoreduced set. Then $\boldsymbol{A}$ is coherent if and only if $\boldsymbol{A}$ is a characteristic set for a prime d.i. of $R$.

\section{The SPecialization theorems}

1. Extension of specializations. In this section we prove the following theorem $\left({ }^{7}\right)$ :

Theorem 1. Let $\left(\xi_{1}, \cdots, \xi_{n}\right)$ be a point, and $N$ a d.p. in R such that $N\left(\xi_{1}, \cdots, \xi_{n}\right) \neq 0 ;$ let $1 \leqq i \leqq n$. Then there exists a d.p. $N_{i}$ in $\mathcal{F}\left\{y_{1}, \cdots, y_{i}\right\}$, with $N_{i}\left(\xi_{1}, \cdots, \xi_{i}\right) \neq 0$, such that any specialization $\left(\eta_{1}, \cdots, \eta_{i}\right)$ of $\left(\xi_{1}, \cdots, \xi_{i}\right)$ over $\mathfrak{F}$ for which $N_{i}\left(\eta_{1}, \cdots, \eta_{i}\right) \neq 0$ can be extended to a specialization $\left(\eta_{1}, \cdots, \eta_{n}\right)$ of $\left(\xi_{1}, \cdots, \xi_{n}\right)$ over $\mathcal{F}$ for which $N\left(\eta_{1}, \cdots, \eta_{n}\right) \neq 0$.

To facilitate the proof, it is convenient to restate the theorem in an equivalent form. To this end, let $\Pi$ be the prime d.i. consisting of those d.p. of $R$ which vanish at the point $\left(\xi_{1}, \cdots, \xi_{n}\right)$ (i.e.: the prime d.i. of $\mathbb{R}$ with generic zero $\left.\left(\xi_{1}, \cdots, \xi_{n}\right)\right)$. In particular, $N\left(\xi_{1}, \cdots, \xi_{n}\right) \neq 0$ means just: $N \notin \Pi$. The set of d.p. of $\mathcal{F}\left\{y_{1}, \cdots, y_{i}\right\}$ which vanish at $\left(\xi_{1}, \cdots, \xi_{i}\right)$ is evidently just $\Pi \cap \mathcal{F}\left\{y_{1}, \cdots, y_{i}\right\}$. Finally, "specialization of a point" is the same thing as "zero of the prime d.i. having the point for generic zero." Hence in these terms Theorem 1 becomes:

Theorem $1^{\prime}$. Let $\Pi$ be a prime d.i. of $R$; let $N \in R, \notin I$; let $1 \leqq i \leqq n$. Then there exists a d.p. $N_{i}$, in $\mathscr{F}\left\{y_{1}, \cdots, y_{i}\right\}$ but not in $\Pi$, such that any zero $\left(\eta_{1}, \cdots, \eta_{i}\right)$ of $\Pi \cap \mathcal{F}\left\{y_{1}, \cdots, y_{i}\right\}$ not annihilating $N_{i}$ can be extended to a zero $\left(\eta_{1}, \cdots, \eta_{n}\right)$ of $\Pi$ not annihilating $N$.

We preface the proof with two major simplifications:

(a) It suffices to prove the case $i=n-1$-for, if we could find an $N_{n-1}$ (as in the theorem) "permitting extensibility" to a zero of $\Pi$ not annihilating $N$, we could then find an analogous $N_{n-2}$ permitting extensibility to a zero

(7) This is the differential-algebraic "lifting" of Lamma 1 of Chevalley [1]. 
of $\Pi \cap \mathcal{F}\left\{y_{1}, \cdots, y_{n-1}\right\}$ not annihilating $N_{n-1}$, and so on (induction on $n-i$ ). (Below we abbreviate $\mathcal{F}\left\{y_{1}, \cdots, y_{n-1}\right\}$ by $\mathbb{R}_{n-1} ; \Pi \cap \mathbb{R}_{n-1}$ by $\Pi_{n-1}$.)

(b) If $\boldsymbol{A}$ is a characteristic set for $\Pi$ (in some given ranking), it suffices to prove the case where $N$ is PR with respect to $A$-for, evidently the partial remainder of an arbitrary $N \notin \Pi$ with respect to $A$ is still $\notin \Pi$, and the $N_{n-1}$ of the theorem which by hypothesis exists for this partial remainder will obviously serve even for the original $N\left(^{8}\right)$.

For the completion of the proof, choose a ranking in which every $y_{n}$ derivative is higher than every $y_{i}$-derivative $(i<n)$; let $A$ be a characteristic set for $\Pi$ in this ranking. Let $v_{1}, \cdots, v_{t}$ be those $y$-derivatives effectively present in $N$ or in a term of $A$; let $v_{1}, \cdots, v_{s}$ be those $v^{\prime}$ s which are not $y_{n}$ derivatives. Let $\mathbb{R}^{\circ}=\mathcal{F}\left[v_{1}, \cdots, v_{t}\right] ; R_{n-1}^{\circ}=\mathcal{F}\left[v_{1}, \cdots, v_{s}\right]\left(=\mathbb{R}^{\circ} \cap R_{n-1}\right)$. Let $\Pi^{\circ}=\Pi \cap R^{\circ} ; \Pi_{n-1}^{\circ}=\Pi \cap R_{n-1}^{\circ}\left(=\Pi^{\circ} \cap \Omega_{n-1}=\Pi^{\circ} \cap R_{n-1}^{\circ}\right)$. Note that $\Pi^{\circ}$ is a prime ideal of $\mathbb{R}^{\circ}$ and $\Pi_{n-1}^{\circ}$ a prime ideal of $\mathcal{R}_{n-1}^{\circ}$; note also that $N, I, S \in \mathbb{R}^{\circ}$ but $\notin \Pi^{\circ}$, while $A \subset \Pi^{\circ}$.

By the algebraic-geometry analog of the present theorem, then, there

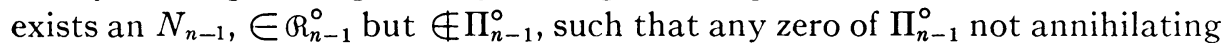
$N_{n-1}$ can be extended to a zero of $\Pi^{\circ}$ not annihilating $I S N$.

Now regard everything as reembedded in $R$; then the choice of $N_{n-1}$ just made implies in particular: If $\left(\eta_{1}, \cdots, \eta_{n-1}\right)$ is a zero of $\Pi_{n-1}$ not annihilating $N_{n-1}$ (which of course $\in \mathbb{R}_{n-1}$ but $\notin \Pi_{n-1}$ ), and we regard it as an ordinary zero of $\Pi_{n-1}$, then it can be extended to an ordinary zero of $\boldsymbol{A}$ (in fact: of $\Pi^{\circ}$ ) not annihilating $I S N$. (In fact: The extension is effected by assigning certain values (in $\Omega$ ) to $v_{s+1}, \cdots, v_{t}$, and then taking for the remaining $y_{n}$-derivatives, say, a set of new transcendental quantities of $\Omega$.) In other words, we have shown that there exists an $N_{n-1}$, in $\mathcal{R}_{n-1}$ but not in $\Pi_{n-1}$, such that if $\left(\eta_{1}, \cdots, \eta_{n-1}\right)$ is any zero of $\Pi_{n-1}$ not annihilating $N_{n-1}$, then, working in the ring $\mathcal{F}\left\langle\eta_{1}, \cdots, \eta_{n-1}\right\rangle\left\{y_{n}\right\}$, and denoting the substitution of $\eta_{1}, \cdots, \eta_{n-1}$ for $y_{1}, \cdots, y_{n-1}$ in a subset of $R$ by the superscript *, we have $I^{*} S^{*} N^{*} \notin\left\langle A^{*}\right\rangle$.

One sees that (the nonzero terms of) $A^{*}$ comprise a coherent autoreduced set in $\mathcal{F}\left\langle\eta_{1}, \cdots, \eta_{n-1}\right\rangle\left\{y_{n}\right\}$ (in the ranking "inherited" from $R$ ). In fact, since we had $I^{*} \neq 0$, these terms have the same leaders (and degrees in them) as their originals in $\boldsymbol{A}$ (which are of course just those $A$ 's with $y_{n}$-derivatives for leaders); the needed properties of $A^{*}$ thus follow naturally from the corresponding properties of this subset of $A$.

Suppose $I^{*} S^{*} N^{*}$ were in $\left\{A^{*}\right\}$, so that $\left(I^{*} S^{*} N^{*}\right)^{\infty} \in\left[A^{*}\right]$. Since $A^{*}$ is coherent autoreduced, and $I^{*} S^{*} N^{*}$ (whence any power thereof) is PR with respect to it, this implies by the lemma that $\left(I^{*} S^{*} N^{*}\right)^{\infty} \in\left(A^{*}\right)$. Hence $I^{*} S^{*} N^{*} \in\left\langle A^{*}\right\rangle$; contradiction.

(8) Without extra effort we could have shown that it even suffices to prove the case where $N$ is reduced with respect to $A$; but the above is all we need. 
We have thus shown that our choice of $\left(\eta_{1}, \cdots, \eta_{n-1}\right)$ actually implies $I^{*} S^{*} N^{*} \in\left\{A^{*}\right\}$. But this just means that there exists an $\eta_{n}$ (in $\Omega$ ) such that $\left(\eta_{1}, \cdots, \eta_{n}\right)$ annihilates $A$ but not ISN-i.e., that $\left(\eta_{1}, \cdots, \eta_{n-1}\right)$ can be extended to a zero of $A$ not annihilating $I S N$. Since $\Pi \subset[A]:(I S)^{\infty}$, this extended zero is actually a zero of $\Pi$, and the theorem is proved.

In algebraic geometry one has the stronger theorem (Weil $[9$, p. 31, Theorem 6]) that any specialization is extensible provided "infinity" is permitted as a value. This is false for differential algebra; the following counterexample is due to Dr. Kolchin: Working in $\mathcal{F}\{y, z\}$ (where $\mathcal{F}$ is an ordinary differential field with derivation denoted by '), let $(\eta, \zeta)$ be a generic zero of the general component of $y z^{\prime 2}+F(z)$, where $F(z)$ is a cubic polynomial in $z$ with constant coefficients and with distinct roots; then the specializaton $\eta \rightarrow 0$ is inextensible.

If $N=1$ in Theorem 1 , it can be seen that we have proved: Let $\Pi$ be $a$ prime d.i. of $R, A$ a characteristic set for $\Pi$ (in a ranking of the type chosen above). Let $\left(\eta_{1}, \cdots, \eta_{n-1}\right)$ be a zero of $\Pi_{n-1}$ which, if regarded (partwise) as an ordinary zero of $\Pi_{n-1}^{\circ}$, is extensible to an ordinary zero of $\Pi^{\circ}$ not annihilating IS. Then $\left(\eta_{1}, \cdots, \eta_{n-1}\right)$ can be extended to a zoro of $\Pi$ not annihilating IS.

Unfortunately, the "IS" cannot be struck out from both sentences in this statement. (Example: Any case where $\Pi^{\circ *}$ properly $\subset \mathcal{F}\left\langle\eta_{1}, \cdots, \eta_{n-1}\right\rangle$ $\cdot\left[v_{s+1}, \cdots, v_{t}\right]$ but $\left.\Pi^{*}=\mathcal{F}\left\langle\eta_{1}, \cdots, \eta_{n-1}\right\rangle\left\{y_{n}\right\}.\right)$ Furthermore, while with the "IS" in the conclusion, the "IS" in the hypothesis is certainly necessary, it is by no means a necessary condition if the "IS" is struck out from the conclusion. (Example: $\Pi_{n-1}=(0), I S \in R_{n-1},[\Pi, I S]$ properly $\subset R,\left(\eta_{1}, \cdots, \eta_{n-1}\right)$ the first part of a zero of a prime d.i. component of $\{\Pi, I S\}$.)

2. Intermediate specializations. In this section we prove a differentialalgebraic analog of the following theorem of algebraic geometry $\left({ }^{9}\right)$.

Let $\left(\xi_{1}, \cdots, \xi_{n}\right) \rightarrow\left(\zeta_{1}, \cdots, \zeta_{n}\right)$ be a specialization $\left({ }^{10}\right)$ over the field $K$, and let (say) $\xi_{1}, \cdots, \xi_{d}$ be a transcendence base for $K\left(\xi_{1}, \cdots, \xi_{n}\right)$ over $K$. Suppose that, for each $d<i \leqq n$, there is an $f_{i} \in K\left[y_{1}, \cdots, y_{i}\right]$ such that $f_{i}\left(\xi_{1}, \cdots, \xi_{i}\right)=0$ and $f_{i}\left(\zeta_{1}, \cdots, \zeta_{i-1}, y_{i}\right) \neq 0$. Let $0 \leqq e \leqq d$. Then any specialization $\left(\eta_{1}, \cdots, n_{e}\right) \rightarrow\left(\zeta_{1}, \cdots, \zeta_{e}\right)$ over $K$ can be extended to an "intermediate specialization" $\left(\xi_{1}, \cdots, \xi_{n}\right) \rightarrow\left(\eta_{1}, \cdots, \eta_{n}\right) \rightarrow\left(\zeta_{1}, \cdots, \zeta_{n}\right)$ over $K$.

The analogous theorem which we shall prove for differential algebra requires a specific choice for the analogs of the $f^{\prime} s\left({ }^{11}\right)$ :

( ${ }^{9}$ ) This follows readily from Proposition 12 of Weil $[9$, p. 65], and in turn implies the more usual intermediate specialization theorem for which "integrality" is required. Weil's Proposition 12 does not itself "lift" to differential algebra; a counterexample can be constructed from the example studied in Ritt [6, p. 133].

(10) In this section the term "specialization" refers to the $m a p \xi \rightarrow \zeta$ rather than to the image, $\zeta$, of $\xi$ under the map as in $\$ 1$. However, no confusion should result.

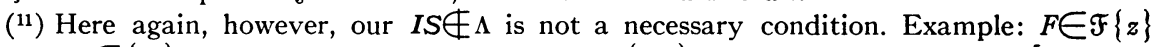
such that $F \in\{S\}$ (where $S$ is the separant of $F$ ); $\Pi=\{\theta F\}$ for suitable $\theta$ (cf. Hillman [2, p. 166, Theorem X]); $\Lambda$ any component of $\{S\}$; work in $\mathbb{R}=\mathfrak{F}\{y, z\}$. 
THEOREM 2. Let $\left(\xi_{1}, \cdots, \xi_{n}\right) \rightarrow\left(\zeta_{1}, \cdots, \zeta_{n}\right)$ be a specialization over $\mathcal{F}$; let II, $\Lambda$ be the prime d.i. of $\mathbb{R}$ with generic zeros $\left(\xi_{1}, \cdots, \xi_{n}\right),\left(\zeta_{1}, \cdots, \zeta_{n}\right)$ respectively. Suppose that there exists an unmixed ranking on $R$ in which $\Pi$ has a characteristic set $\boldsymbol{A}$ such that $I S \notin \Lambda$. Without loss of generality, we can assume that in this ranking $y_{1}, \cdots, y_{d}$ (where $d=\operatorname{dim} \Pi$ ) are parametric indeterminates for $\Pi$. Let $0 \leqq e \leqq d$. Then any specialization $\left(\eta_{1}, \cdots, \eta_{e}\right) \rightarrow\left(\zeta_{1}, \cdots, \zeta_{e}\right)$ over $\mathfrak{F}$ can be extended to an "intermediate specialization" $\left(\xi_{1}, \cdots, \xi_{n}\right)$ $\rightarrow\left(\eta_{1}, \cdots, \eta_{n}\right) \rightarrow\left(\zeta_{1}, \cdots, \zeta_{n}\right)$ over $\mathcal{F}$.

As in the last section, it is convenient to translate the statement of the theorem from the terminology of specializations of points into that of zeros of prime d.i. To complete this translation here we need only replace the first two sentences of the statement of the theorem by "Let $\Pi \subset \Lambda$ be prime d.i. of $R$," and the last sentence by "Then any point $\left(\eta_{1}, \cdots, \eta_{e}\right)$ which annihilates nothing in $\mathfrak{F}\left\{y_{1}, \cdots, y_{e}\right\}$ outside $\Lambda$ can be extended to a zero $\left(\eta_{1}, \cdots, \eta_{n}\right)$ of $I I$ which annihilates nothing in $R$ outside $\Lambda$."

(A specialization $\left(\xi_{1}, \cdots, \xi_{n}\right) \rightarrow\left(\zeta_{1}, \cdots, \zeta_{n}\right)$ satisfying the hypothesis of the Theorem will be called proper (with respect to the given ranking); similarly, if $\Pi \subset \Lambda$ are prime d.i. of $R$ satisfying the hypothesis of the theorem, $\Lambda$ will be called nonsingular over $\Pi$ (with respect to this ranking). More generally, given a ranking on $R$ and an autoreduced set $A \subset R, \Lambda \subset R$ will be called nonsingular over $A$ if $A \subset \Lambda$ and $I S \notin \Lambda$.)

We preface the proof with two simplifying observations:

(a) It suffices to prove the case $e=d$, since extension "from $e$ to $d$ " can always be effected by simply taking as $\eta_{e+1}, \cdots, \eta_{d}$ a set of differentially algebraically independent quantities (of $\Omega$ ).

(b) As observed for Theorem 1 , it suffices to show that $\left(\eta_{1}, \cdots, \eta_{d}\right)$ is extensible to a zero of $I$ which annihilates nothing outside $\Lambda$ which is PR with respect to $A$.

Let, then, $G \in R, \notin \Lambda$ be PR with respect to $A$. Let $t_{1}, \cdots, t_{s}$ be those $y$-derivatives other than $u_{1}, \cdots, u_{k}$ which are effectively present in a term of $A$ or in $G$; let $\mathbb{R}^{\circ}=\mathcal{F}\left[t_{1}, \cdots, t_{s}, u_{1}, \cdots, u_{k}\right], \Pi^{\circ}=\Pi \cap R^{\circ}, \Lambda^{\circ}=\Lambda \cap R^{\circ}$. Let $R^{t}=\mathscr{F}\left[t_{1}, \cdots, t_{s}\right], \Lambda^{t}=\Lambda \cap R^{t}$. Clearly $\Pi^{\circ} \cap R^{t}=(0)$; from this and from $I \notin \Lambda^{\circ}$ it follows, by the theorem of algebraic geometry stated at the beginning of this section, that any point $\left(\rho_{1}, \cdots, \rho_{s}\right)$ which annihilates nothing in $\mathbb{R}^{t}$ outside $\Lambda^{t}$ can be extended to a zero $\left(\rho_{1}, \cdots, \rho_{s}, \tau_{1}, \cdots, \tau_{k}\right)$ of $\Pi^{\circ}$ which annihilates nothing in $\mathbb{R}^{\circ}$ outside $\Lambda^{\circ}$.

Let $\mathcal{F}\left\{y_{1}, \cdots, y_{d}\right\}=\mathfrak{R}_{d}, \Lambda \cap \mathfrak{R}_{d}=\Lambda_{d}$. (Clearly $\Pi \cap \mathfrak{R}_{d}=(0)$.) Let $\mathfrak{R}_{d}^{0}$ $=\mathbb{R}^{\circ} \cap \mathfrak{R}_{d}, \Lambda_{d}^{\circ}=\Lambda \cap \mathfrak{R}_{d}^{\circ}$. Evidently $\mathcal{R}_{d}^{\circ}$ is just $\mathcal{F}\left[t_{1}, \cdots, t_{r}\right]$ for some $r \leqq s$. We are given a point $\left(\eta_{1}, \cdots, \eta_{d}\right)$ which annihilates nothing in $R_{d}$ outside $\Lambda_{d}$. Let the values which this point assigns to the $y$-derivatives $t_{1}, \cdots, t_{r}$ be $\rho_{1}, \cdots, \rho_{r} ;\left(\rho_{1}, \cdots, \rho_{r}\right)$ is thus a point which annihilates nothing in $\mathcal{R}_{d}^{0}$ outside $\Lambda_{d}^{\circ}$. If we then let $\rho_{r+1}, \cdots, \rho_{s}$ be new transcendental quantities of $\Omega$, 
we obtain a point $\left(\rho_{1}, \cdots, \rho_{s}\right)$ which annihilates nothing in $\mathcal{R}^{t}$ outside $\Lambda^{t}$.

Now reembed in $R$ and "extend" $\left(\eta_{1}, \cdots, \eta_{d}\right)$ by letting $t_{r+1}, \cdots, t_{s}$, $u_{1}, \cdots, u_{k}$ go into $\rho_{r+1}, \cdots, \rho_{s}, \tau_{1}, \cdots, \tau_{k}$ as in the last two paragraphs, and the remaining $y$-derivatives into new differentially transcendental quantities of $\Omega$; the result is (in particular) an ordinary zero of $\boldsymbol{A}$ which does not annihilate $I S G$. If we denote the substitution of $\eta_{1}, \cdots, \eta_{d}$ for $y_{1}, \cdots, y_{d}$ by the superscript ${ }^{*}$, we see that we have thus shown: $G \notin \Lambda, \mathrm{PR}$ with respect to $A \Rightarrow I^{*} S^{*} G^{*} \notin\left\langle A^{*}\right\rangle$. As in the proof of Theorem I, this implies that $I^{*} S^{*} G^{*} \notin\left\{A^{*}\right\}$. It follows that some prime d.i. component $\Upsilon$ of $\left\{A^{*}\right\}$ (in $\left.\mathcal{F}\left\langle\eta_{1}, \cdots, \eta_{d}\right\rangle\left\{y_{d+1}, \cdots, y_{n}\right\}\right)$ must fail to contain $G^{*}$ for any such $G$. Let $\left(\eta_{d+1}, \cdots, \eta_{n}\right)$ be a generic zero of $\Upsilon$; then $\left(\eta_{1}, \cdots, \eta_{n}\right)$ is a zero of $A$ which annihilates no such $G$. Since $I S$ is such a $G$, and $\Pi \subset[A]:(I S)^{\infty}$, this $\left(\eta_{1}, \cdots, \eta_{n}\right)$ is actually a zero of $\Pi$, and the theorem is proved.

\section{Applications}

\section{Chain theorems.}

Proposition 1. Let $\Pi \subset \mathbb{Q}$ be a prime d.i. of dimension $d$; let $0 \leqq e<d$; let $F \in R, \notin \Pi$. Then there exists a prime d.i. $\Lambda \subset R$, of dimension $e$, such that $\Pi \subset \Lambda$ and $F \notin \Lambda\left({ }^{12}\right)$.

Proof. By induction on $d-e$, it suffices to prove the case $e=d-1$. Rank the $y$ 's so $\Pi \cap \mathcal{F}\left\{y_{1}, \cdots, y_{d}\right\}=(0)$. Choose $G \in \mathcal{F}\left\{y_{1}, \cdots, y_{d}\right\}$, as in Theorem 1 , so any zero of $\Pi \cap \mathcal{F}\left\{y_{1}, \cdots, y_{d}\right\}$ not annihilating $G$ can be extended to a zero of $\Pi$ not annihilating $I F$ (where $I$ is the product of the initials for some characteristic set for $\Pi$ in the given ranking). Let $v$ be a proper derivative of the leader of $G$; let $\left(\eta_{1}, \cdots, \eta_{d}\right)$ be a generic zero of the prime d.i. [v] $\mathcal{C F}\left\{y_{1}, \cdots, y_{d}\right\}$. Then $\left(\eta_{1}, \cdots, \eta_{d}\right)$ has dimension $d-1$ and does not annihilate $G$. It follows by Theorem 1 that $\left(\eta_{1}, \cdots, \eta_{d}\right)$ can be extended to a zero $\left(\eta_{1}, \cdots, \eta_{n}\right)$ of $\Pi$ which does not annihilate $I F$; this last implies in particular that $\left(\eta_{1}, \cdots, \eta_{n}\right)$ still has dimension $d-1$. Take as $\Lambda$ the prime d.i. of $R$ with generic zero $\left(\eta_{1}, \cdots, \eta_{n}\right)$, and the proof is complete.

PROPOSITION 2. Let $\Pi \subset \Lambda$ be prime d.i. of $R$ of dimensions $d$, e respectively, such that $\Lambda$ is nonsingular over $\Pi$ with respect to some unmixed ranking. Let $e<i<d$. Then there is a prime d.i. $\Upsilon \subset \mathcal{R}$, of dimension $i$, contained between $\Pi$ and $\Lambda$.

Proof. Without loss of generality, we can assume that the given ranking is such that $\Pi \cap \mathcal{F}\left\{y_{1}, \cdots, y_{d}\right\}=(0)$ (i.e., that $y_{1}, \cdots, y_{d}$ are parametric indeterminates for $\Pi$ in this ranking). Since $I$ (defined as in the proof of the preceding proposition) $\notin \Lambda, \Lambda_{d}=\Lambda \cap F\left\{y_{1}, \cdots, y_{d}\right\}$ still has dimension $e$.

(12) This proposition is contained in the Hilbert's Nullstellensatz (strong form) of Seidenberg [7]. The present proof is somewhat more direct. (The author wishes to thank the referee for calling his attention to this reference.) 
One can easily construct an $\Upsilon_{d} \subset \Lambda_{d}$ of dimension $i$ (e.g., use the d.i. generated in $\mathcal{F}\left\{y_{1}, \cdots, y_{d}\right\}$ by the intersection of $\Lambda_{d}$ with some suitable $\left.\mathcal{F}\left\{y_{1}, \cdots, y_{j}\right\}\right)$. By Theorem 2, $\Upsilon_{d}$ is the intersection with $\mathcal{F}\left\{y_{1}, \cdots, y_{d}\right\}$ of some prime d.i. $\Upsilon \subset R$ contained between $\Pi$ and $\Lambda$; since $\Lambda$-and so a fortiori $\Upsilon$-is nonsingular over $\Pi, \Upsilon$ must still have dimension $i$, which completes the proof.

Proposition 3. Let $\Pi \subset \Lambda$ be prime d.i. of $R$ of dimensions $d, d-1$ respectively, such that $\Lambda$ is nonsingular over $\Pi$ I with respect to some unmixed ranking. Then there exists an infinite descending chain of prime d.i., all of dimension $d-1$, between $\Lambda$ and $\Pi$.

Proof. Let the ranking be as in the proof of Proposition 2. Since $\operatorname{dim} \Lambda$ $=d-1$, we have $\Lambda_{d}=\Lambda \cap \mathcal{F}\left\{y_{1}, \cdots, y_{d}\right\} \neq(0)$. Let $F$ be any irreducible d.p. in $\Lambda_{d}$. By Theorem VIII of Hillman [2, p. 166], one can construct an infinite strictly descending chain $\Upsilon_{d}^{(1)} \supset \Upsilon_{d}^{(2)} \supset \ldots \supset(0)$ consisting of components of successive derivatives of $F$. In such a chain, let $\Upsilon_{d}^{(1)}$ be a component of $F$ which is contained in $\Lambda_{d}$. By Theorem $2, \Upsilon_{d}^{(1)}$ is the intersection with $F\left\{y_{1}, \cdots, y_{d}\right\}$ of a prime d.i. $\Upsilon^{(1)} \subset \Omega$ which is contained between II and $\Lambda$; evidently $\Upsilon^{(1)}$ has dimension $d-1$. Since $\Upsilon^{(1)}$ is a fortiori nonsingular over $\Pi$, by Theorem 2 again we can "extend" $\Upsilon_{d}^{(2)}$ to a prime d.i. $\Upsilon^{(2)} \subset \propto$ contained between $\Pi$ and $\Upsilon^{(1)}$ and with dimension $d-1$. Since all these inclusions are evidently proper, iteration of this argument completes the proof.

\section{A theorem on dimensions.}

THEOREM 3. With respect to a given unmixed ranking on $R$, let $A \subset \Omega$ be a coherent autoreduced set for which the number of parametric indeterminates is $d$; let $\Lambda$ be a nonsingular component of $\{\boldsymbol{A}\}$. Then $\operatorname{dim} \Lambda=d$.

Proof. Without loss of generality, we can assume that $y_{1}, \cdots, y_{d}$ are parametric indeterminates for $\boldsymbol{A}$. Let $Q$ be a minimal prime ideal divisor of $\boldsymbol{A}$ contained in $\Lambda$. Evidently $Q$ is generated in $R$ by d.p. involving only those $y$-derivatives which occur in the terms of $A$; suppose there are $N$ of these and look at the restriction of $Q$ to the polynomial ring which they generate over $\mathcal{F}$. By algebraic geometry, it follows that ord $Q \geqq N-k$ (where $k$ is the number of terms in $A$ ). But since $A \subset Q$ and $I \notin Q$, ord $Q$ cannot exceed this, hence must equal it. Thus a generic zero for $Q$ puts quantities which are algebiaically independent over $\mathcal{F}$ for all the $y$-derivatives other than the $u$ 's (=the leaders of the terms of $\boldsymbol{A}$ ).

Let $\xi_{1}, \cdots, \xi_{d}$ be elements of $\Omega$ which are differentially algebraically independent over $\mathcal{F}$; then by what we have just seen, this set of elements taken together with all their derivatives has an extension to an ordinary zero of $\boldsymbol{A}$ which annihilates nothing outside $Q$-namely: to a generic zero of $Q$. In particular, $\left(\xi_{1}, \cdots, \xi_{d}\right)$ has an extension to an ordinary zero of $A$ which annihilates nothing which is outside $\Lambda$ and PR with respect to $A$.

By an argument used in the proof of Theorem 2, it follows that 
$\left(\xi_{1}, \cdots, \xi_{d}\right)$ must have an extension to a ("differential") zero $\left(\xi_{1}, \cdots, \xi_{n}\right)$ of $\boldsymbol{A}$ which annihilates nothing outside $\Lambda$.

Let $\Pi$ be the prime d.i. of $R$ with generic zero $\left(\xi_{1}, \cdots, \xi_{n}\right)$. Then $A \subset \Pi \subset \Lambda$, so that by the minimality of $\Lambda$ over $A$ we must have $\Pi=\Lambda$. This means that a generic zero of $\Lambda$ (namely: $\left(\xi_{1}, \cdots, \xi_{n}\right)$ ) has its first $d$ coordinates differentially algebraically independent over $\mathcal{F}$, so that $\operatorname{dim} \Lambda \geqq d$. But since $A \subset \Lambda$ and $I \notin \Lambda$, we must have $\operatorname{dim} \Lambda \leqq d$. Combining these two inequalities completes the proof.

3. Intersection theory. Ritt [6, beginning of Chapter VIII] has shown by example that for differential algebra it is not always true that the components of the intersection of two varieties (in $\Omega^{n}$ ) of dimensions $r$ and $s$ must have dimensions at least $r+s-n$.

In this section we apply the theorems of the present paper to prove some special results on the dimensions of the components of an intersection. For convenience, we work with the prime d.i. rather than with the corresponding irreducible varieties.

Proposition 4. Let $\Pi$, $\Upsilon$ be prime d.i. of R of dimensions $r$, s. Let $\left(\xi_{1}, \cdots, \xi_{n}\right)$ be a generic zero for $\Pi$. Suppose that $\Upsilon$ is generated in $\mathbb{R}$ by d.p. which involve only derivatives of $(s a y) y_{i_{1}}, \cdots, y_{i_{k}}$, and that $\xi_{i_{1}}, \cdots, \xi_{i_{k}}$ are differentially algebraically independent over $\mathcal{F}$. Embed $\xi_{i_{1}}, \cdots, \xi_{i_{k}}$ into a differential transcendence base $\xi_{i_{1}}, \cdots, \xi_{i_{r}}$ for $\mathcal{F}\left\langle\xi_{1}, \cdots, \xi_{n}\right\rangle$ over $\mathcal{F}$. Choose an unmixed ranking on $\mathbb{R}$ in which $y_{i_{1}}, \cdots, y_{i_{r}}$ are parametric indeterminates for $\Pi$. With respect to this ranking, let $\Lambda$ be a component of $\{\Pi, \Upsilon\}$ which is nonsingular over $\Pi$. Then $\operatorname{dim} \Lambda=r+s-n$.

Proof. Without loss of generality we may suppose that the parametric indeterminates are $y_{1}, \cdots, y_{r}$. We have $\Upsilon \cap \mathcal{F}\left\{y_{1}, \cdots, y_{r}\right\} \subset \Lambda$ $\cap F\left\{y_{1}, \cdots, y_{r}\right\}$; hence by Theorem 2 , the former is the intersection with $\mathcal{F}\left\{y_{1}, \cdots, y_{r}\right\}$ of some prime d.i. $\Xi$ of $R$ such that $\Pi \subset \Xi \subset \Lambda$. Since $\Xi$ is a fortiori nonsingular over $\Pi$, we have $\operatorname{dim} \Xi=\operatorname{dim} \Upsilon \cap \mathcal{F}\left\{y_{1}, \cdots, y_{r}\right\}$ $=s-(n-r)=r+s-n$. But evidently $\Pi, \Upsilon$ each $\subset$ 寻, so that (by minimality of $\Lambda$ over $\{\Pi, \Upsilon\}$ ) we must have $\Xi=\Lambda$; this completes the proof.

Proposition 5. Let $\Pi, \Upsilon$ be prime d.i. of R of dimensions $r$, s. Let $\left(\xi_{1}, \cdots, \xi_{n}\right)$ be a generic zero for $\Pi$. Suppose that $\Upsilon$ is generated in $R$ by d.p. which involve only derivatives of $\left(\right.$ say) $y_{i_{1}}, \cdots, y_{i_{k}}$, and that $\xi_{i_{1}}, \cdots, \xi_{i_{k}}$ have dimension $\leqq n-s$ over $\mathcal{F}$. Embed a differential transcendence base of these $\xi$ 's for $\mathcal{F}\left\langle\xi_{i_{1}}, \cdots, \xi_{i_{k}}\right\rangle$ over $\mathcal{F}$ into a differential transcendence base $\xi_{j_{1}}, \cdots, \xi_{j_{r}}$ for $\mathcal{F}\left\langle\xi_{1}, \cdots, \xi_{n}\right\rangle$ over $\mathcal{F}$. Choose an unmixed ranking on $\mathbb{R}$ in which $y_{j_{1}}, \cdots, y_{j_{r}}$ are parametric indeterminates for $\Pi$, and in which those of $y_{i_{1}}, \cdots, y_{i_{k}}$ which are not parametric indeterminates for $\Pi$ rank lower than all the other nonparametric $y$ 's. With respect to this ranking, let $\Lambda$ be a component of $\{\Pi, \Upsilon\}$ which is nonsingular over $\Pi$. Then $\operatorname{dim} \Lambda \geqq r+s-n$. 
Proof. Without loss of generality, let the parametric indeterminates be $y_{1}, \cdots, y_{r}$. Let $\boldsymbol{A}$ be a characteristic set for $\Pi$ in the given ranking for which $I S \notin \Lambda$. It can be seen that the substitution of $\xi_{i_{1}}, \cdots, \xi_{i_{k}}$ for $y_{i_{1}}, \cdots, y_{i_{k}}$ and hence $a$ fortiori the substitution of $\zeta_{i_{1}}, \cdots, \zeta_{i_{k}}$ for $y_{i_{1}}, \cdots, y_{i_{k}}$ (where $\zeta_{1}, \cdots, \zeta_{n}$ is a generic zero for $\Lambda$ )-must annihilate all the terms of $A$ whose leaders are $y_{i_{j}}$-derivatives $(1 \leqq j \leqq k)$. Furthermore, since $I \notin \Lambda$, the remaining terms of $A$ are still a coherent autoreduced set even after the latter substitution. If we work in $\mathcal{F}\left\langle\zeta_{i_{1}}, \cdots, \zeta_{i_{k}}\right\rangle\left\{y_{h_{1}}, \cdots, y_{h_{n-k}}\right\}$ (where these last are the $y$ 's other than $y_{i_{1}}, \cdots, y_{i_{k}}$ ), and denote the substitution of $\zeta_{i_{1}}, \cdots, \zeta_{i_{k}}$ for $y_{i_{1}}, \cdots, y_{i_{k}}$ by the superscript ${ }^{*}$, it can be seen that $\Lambda^{*}$ is a component of (the set of nonzero terms of) $\boldsymbol{A}^{*}$ and that it does not contain $I^{*} S^{*}$ (in fact, that these terms of $A^{*}$ are a characteristic set for $\left.\Lambda^{*}\right)$. It follows by Theorem 3 that $\operatorname{dim} \Lambda^{*}=$ number of parametric indeterminates for $A^{*} \geqq(n-k)$ $-((n-r)-(k-(n-s)))=r+s-n$. Finally, since $\zeta_{h_{1}}, \cdots, \zeta_{h_{n-k}}$ is a generic zero for $\Lambda^{*}$, we have $\operatorname{dim} \Lambda \geqq \operatorname{dim} \Lambda^{*}$; this completes the proof.

Propositions 4 and 5 add up to a theorem on the dimensions of certain "strongly nonsingular" components of the perfect d.i. generated by the sum of two prime d.i. As a preliminary to this, let us observe that, just as in algebraic geometry, if $V, W \subset \Omega^{n}$ are irreducible varieties of dimensions $r, s$ over $\mathcal{F}$, then the Cartesian product $V \times W \subset \Omega^{2 n}$ is a variety every component of which has dimension $r+s$ over $\mathcal{F}$. (The proof makes use of the fact that if $\mathcal{F}$ is algebraically closed, $V \times W$ is an irreducible variety; see Kolchin [3, p. 769]). Now there is a natural one-to-one dimension-preserving correspondence between the components of $V \cap W$ and those of $(V \times W) \cap \Delta$ (where $\Delta$ is the "diagonal" of $\Omega^{2 n}$ regarded as $\Omega^{n} \times \Omega^{n}$ ). Since $\Delta$ is the irreducible variety corresponding to the prime d.i. $\Gamma=\left[z_{1}-y_{1}, \cdots, z_{n}-y_{n}\right] \subset \Omega^{2}$ $=\mathcal{F}\left\{y_{1}, \cdots, y_{n}, z_{1}, \cdots, z_{n}\right\}$, we see that (in a sense) it suffices to prove that if $\Pi$ is a prime d.i. of $\Omega^{2}$ of dimension $d$, then any (sufficiently nonsingular) component of $\{\Pi, \Gamma\} \subset \Re^{2}$ has dimension at least $d-n$.

Let us call $\Lambda \supset \Pi$ absolutely nonsingular over $\Pi$ if it is nonsingular over $\Pi$ and remains so in any unmixed ranking which arises from the given unmixed ranking by permuting the $y$ 's. Let us further call $\Lambda \supset I I$ strongly nonsingular over $\Pi$ if it is absolutely nonsingular over any prime d.i. between $\Pi$ and $\Lambda$.

THEOREM 4. Let $\Pi$ be a prime d.i. of $\mathcal{R}^{2}$ of dimension d; let $\Lambda$ be a component of $\{\Pi, \Gamma\}$ which is strongly nonsingular over $\Pi$. Then $\operatorname{dim} \Lambda \geqq d-n$.

Proof. For $0 \leqq i \leqq n-1$, define $\Pi_{i}$ inductively by $\Pi_{0}=\Pi, \Pi_{i+1}=$ any component of $\left\{\Pi_{i}, z_{i+1}-y_{i+1}\right\}$ which is contained in $\Lambda$. One sees that each $\Pi_{i+1}$ is absolutely nonsingular over $\Pi_{i}$, and that $\Pi_{n}=\Lambda$. Let $\left(\eta_{1}^{(i)}, \cdots, \eta_{n}^{(i)}\right.$, $\left.\zeta_{1}^{(i)}, \cdots, \zeta_{n}^{(i)}\right)$ be a generic zero for $\Pi_{i}$. If $\eta_{i+1}^{(1)}, \zeta_{i+1}^{(i)}$ are differentially algebraically independent over $\mathcal{F}$, then $\operatorname{dim} \Pi_{i+1}=\operatorname{dim} \Pi_{i}-1$ by Proposition 4; but if they are differentially algebraically dependent over $\mathcal{F}$, then $\operatorname{dim} \Pi_{i+1}$ 
$\geqq \operatorname{dim} \Pi_{i}-1$ by Proposition 5. Induction on $n$ thus completes the proof of the theorem.

\section{REFERENCES}

1. C. Chevalley, An algebraic proof of a property of Lie groups, Amer. J. Math. vol. 63 (1941).

2. A. Hillman, On the differential algebra of a single differential polynomial, Ann. of Math. vol. 56 (1952).

3. E. R. Kolchin, Algebraic matric groups and the Picard-Vessiot theory of homogeneous linear ordinary differential equations, Ann. of Math. vol. 49 (1948).

4. - Galois theory of differential fields, Amer. J. Math. vol. 75 (1953). $(1940)$.

5. J. F. Ritt, On a type of algebraic differential manifold, Trans. Amer. Math. Soc. vol. 48

6. - Differential algebra, Amer. Math. Soc. Colloquium Publications, vol. 33, New York, 1950. (1952).

7. A. Seidenberg, Some basic theorems in differential algebra, Trans. Amer. Math. Soc. vol. 73

8. - An elimination theory for differential algebra, Univ. California Publ. Math. (New Series) vol. 3, no. 2, 1956.

9. A. Weil, Foundations of algebraic geometry, Amer. Math. Soc. Colloquium Publications, vol. 29, New York, 1946.

Columbia University,

NEW York, N. Y. 